\title{
Interactive comment on "Latitudinal variation of Pc3-Pc5 geomagnetic pulsation amplitude across the dip equator in central South America" by Graziela Belmira Dias da Silva et al.
}

\section{Graziela Belmira Dias da Silva et al.}

graziela.silva@inpe.br

Received and published: 9 October 2019

The authors gratefully thank to the Referee for the constructive comments and recommendations which definitely help to improve the readability and quality of the manuscript. Following the editors' suggestion, the response is structured as follows: comments from the referee, authors' response, and changes to be made to the manuscript.

Specific comments:

Section 5: To explain the equatorial enhancement of Pc 3 pulsations in the $\mathrm{H}$ compo- 
nent at the dawn terminator, the authors suggested a mechanism that eastward neutral winds in the F-region ionosphere across the dawn terminator cause the enhancement of zonal electric field. This is similar to the generation mechanism for the prereversal enhancement of the zonal electric field during the sunset period. However, this mechanism may explain the enhancement in Sq current around the dawn terminator, but it cannot cause the enhancement in Pc3 amplitude. The authors need to explain how the neutral winds can give rise to the electric field oscillations at the Pc 3 frequency. I guess that this mechanism is difficult to explain the equatorial enhancement of Pc3 amplitude, therefore, the authors should suggest other possible mechanism.

Reply: Analyzing the scientific literature on how ULF wave fields couple to the ionosphere to generate secondary fields modulated by background conductivity during sunrise hours, we are now proposing that this $\mathrm{H}$-component enhancement effect in Pc3 pulsations at the dawn terminator should be mainly related to the mode of propagation and incidence of these waves on the ionosphere. For example, the model of fast mode incidence on the ionosphere described by Alperovich and Fedorov [2007, p. 284-297] predicts that compressional waves in the Pc3 frequency band are those expected to produce the strongest structure of zonal electric fields and currents in the vicinity of the dip equator (mainly during the equinoxes) because its effect increases with increasing frequency. In addition, this model also allows external electric fields other than those carried by the waves (eg, induced by dynamo action) to contribute substantially to increased zonal currents and consequent signal amplification on the ground. We understand that the dynamo mechanism suggested in our text, in which the $F$ region thermo-atmospheric winds drive zonal electric currents, can be applied to explain the Pc3s enhancement near sunrise, given the large longitudinal variations in electrodynamic processes in the South American equatorial ionosphere. However, we still need to assume that our Pc3 events occurring around sunrise are also related to compressional MHD waves vertically incident on the ionosphere, as recognized for the Pc3 events around noon.

ANGEOD

Interactive comment
Printer-friendly version

Discussion paper 
Changes to be made to the manuscript: This discussion will be included in the manuscript, including the summary and conclusions. In addition, a new reference will be added to the text: Alperovich, L.S., Fedorov, E.N.: Hydromagnetic Waves in the Magnetosphere and the lonosphere. Series: Astrophysics and Space Science Library, Vol. 353, 2009, XXIV, 418 pp., doi:10.1007/978-1-4020-6637-5, 2007.

Line 11-15 of page 14: The authors described that the Earth-ionosphere waveguide propagation model is inconsistent with the phase lag of $P_{C} 5$ pulsations at the dip equator. However, according to Shinohara et al. [1998], the phase lag can be explained by the induction effect of the equatorial enhanced ionospheric current above the good conductor Earth. The source of the equatorial ionospheric current can be the electric field propagating from high latitude by the Earth-ionosphere waveguide mode. On the other hand, it is not clear whether or not the MHD wave propagation model can cause the phase lag at the dip equator.

Reply: The reviewer is correct in stating that incident waves coming horizontally from the poles through the Earth-ionosphere waveguide can cause phase delays in magnetic signals at the dip equator relative to off-equatorial regions. This information will be added to our manuscript, as these phase delays can be explained by invoking the induction effect with the model devised by Shinohara et al. [1998]. On the other hand, a discussion on the possibility that the MHD wave propagation model could also cause phase delay in the dip equator had already been presented in our text (lines 9-12 of page 13). We had explained that the screening effect induced by enhanced ionospheric conductivity in the equator is also recognized for causing amplitude depression and phase delays on MHD signals incident from the magnetosphere.

Changes to be made to the manuscript: Corrections will be made to the manuscript to include the induction effect discussed by Shinohara et al. [1998].

Technical corrections:

Line 3 of page 2: internally to the magnetosphere -> internally in the magnetosphere 
Reply/Changes in manuscript: It will be corrected in the text.

Line 4 of page 6: Please describe the period range of bandpass filter for $\mathrm{Pc} 3$ and $\mathrm{Pc}$ ANGEOD 5 pulsations in the text.

Reply/Changes in manuscript: Information will be included in the text.

Line 8-9 of page 8: Please state the duration of the Pc 3 and Pc 5 events. Is the duration fixed? For example, if $\mathrm{Pc} 3$ pulsations continued during 30 minutes, are they counted as one event?

Reply/Changes in manuscript: Most Pc3 events analyzed have duration of about 3 minutes, while Pc5s presented durations shorter than 24 minutes. For all cases, we considered them as a single event, regardless of their duration. This information will be included in the text.

Subsection 3.2: Was the power spectral analysis applied to bandpass-filtered data? I recommend that the authors explain the analysis procedure made by Roy and Rao [1998] briefly.

Reply/Changes in manuscript: Yes, the power spectral analysis was applied to bandpass-filtered data. This information will be included in the text and we will also give more details about the procedure performed by Roy and Rao [1998].

Figure 5, 6, 7: In this manuscript, the amplification factor is defined as the amplitude ratio of pulsations at $\mathrm{CUI}$ to those at other stations, so greater values than one correspond to equatorial depression and smaller values than one correspond to equatorial amplification. However, this definition is a little bit confusing. In addition, different definition is used in Figure 8. Thus, I suggest that the authors use the amplitude ratio of (other stations)/CUI all through the manuscript.

Reply/Changes in manuscript: We accept the referee's suggestion and therefore the definition of the amplification factor (and Figures 6-7) will be changed throughout the manuscript.

Interactive

comment 
Figure 10: Please plot the $H / D$ amplitude ratio or the $P R M(D) / C U I(H)$ ratio in this figure. Reply/Changes in manuscript: The $\mathrm{PRM}(\mathrm{D}) / \mathrm{CUI}(\mathrm{H})$ ratio was obtained for each $\mathrm{Pc} 3$ ANGEOD event and will be plotted in Figure 10 along with the $\mathrm{PRM}(\mathrm{H}) / \mathrm{CUI}(\mathrm{H})$ ratio. Text will be included to discuss the results.Âă

Interactive comment on Ann. Geophys. Discuss., https://doi.org/10.5194/angeo-2019-73,

Interactive

comment 2019. 